\title{
IMPLEMENTATION OF CLOSE RANGE PHOTOGRAMMETRY USING MODERN NON-METRIC DIGITAL CAMERAS FOR ARCHITECTURAL DOCUMENTATION
}

\author{
Mariem A. ELHALAWANI (1) ${ }^{*}$, Zaki M. ZEIDAN ${ }^{2}$, Ashraf A. A. BESHR (iD 3 \\ ${ }^{1}$ Civil Engineering Department, Faculty of Engineering, Mansoura University, 35516 Mansoura, Egypt \\ 2, ${ }^{3}$ Public Works Department, Faculty of Engineering, Mansoura University, 35516 Mansoura, Egypt
}

Received 26 September 2019; accepted 12 March 2021

\begin{abstract}
The development of applied geodetic techniques for mapping and documentation of historical structures, buildings and sites is an important and vital purpose for contribution of any recording of cultural heritage for any country such as Egypt. This is done to preserve and restore any valuable architectural or other cultural monument, as a support to architectural, archaeological and other art-historical research throughout the ages. The purpose of this paper is to use close range photogrammetry technique (CRP) to reconstruct $3 \mathrm{D}$ model of architectural and historical mosque facade and comparing the accuracy of using digital commercial non-metric cameras with different resolutions and metric camera with flatbed scanner and photogrammetric scanner for architectural building documentation. El-Nasr Mosque façade in Mansoura city, Egypt was chosen as a case study in this paper. At first, twenty five points were selected at mosque façade at different elevations and distributed at different façade surfaces and observed using total station. Some of these points were selected as control points and the others were selected as check points to validate the results. Effect of control point's number on image processing and analysis is also studied. Three cameras positions were selected for imaging to get the full details of mosque façade. Close range Digital Workstation (CDW) program was used for processing and analysis of multiple images. The results are indicated that close range photogrammetry using metric camera with photogrammetry scanner instead of flatbed scanner in technique is accurate enough to be beneficial in 3D architectural building documentation. Digital cameras with CRP technique give up different accuracy that depends mainly on the resolution of cameras and camera specifications.
\end{abstract}

Keywords: 2D facade, CRP, metric camera, digital camera, Close range Digital Workstation (CDW) program.

\section{Introduction}

Egypt is the richest country all over the world in terms of archeological sites where there are nearly third of the world's historical sites and buildings in Egypt. Preserving these historical places in good conditions is a national goal since they attract millions of tourists which make a significant contribution to the Egyptian economy (Elsharkawy, 2017).

Digital close range photogrammetry can be considered one of the optimum techniques used for archeological documentation of historical artifacts for ages. Along time, high cost, skills and big effort were required to documenting archaeological sites by traditional studies (Khalaf et al., 2018). So, it's necessary to find an accurate precise technique, at the same time, high resolution Architectural documentation, acquired by digital cameras (Markiewicz et al., 2018). Recently, as a result of improvements of digital photogrammetry, high resolution digital cameras and computer software, reconstruction of buildings as three-dimensional took part amongst current research topics (Suveg \& Vosselman, 2000). The advantage of using digital photogrammetry is its capability of deriving all appropriate measurements from the images itself instead of measurements directly on the objects (Luhmann, 2010). This technology provides substantial reductions in the cost of archaeological surveys and in enhancing survey results. Recent advances in photogrammetric software have led to highly automated workflow (Abed et al., 2017). Also the use of low cost digital photogrammetric systems has contributed to the use of these "off the shelf" cameras among photogrammetrist and non photogrammetrist (Cardenal et al., 2004).

Therefore, it is necessary to study the use of digital cameras in surveying measurements and the reliability of

*Corresponding author. E-mail: mariem_elhalawani@yahoo.com 
accuracy in the documentation and monitoring of buildings.in order to reach the goal of the study, the research explicates façade of one known archaeological mosque, the definition of its history, Types of digital cameras and comparison between the metric camera in terms of accuracy and cost. The data acquisition is concerned with obtaining what may be termed the raw data or raw information (Suveg \& Vosselman, 2000). The raw data is realized in the form of photographs. These photographs can be collected using normal, digital, metric as well as non-metric cameras.

\section{Research objectives}

The main aim of this paper is to study and compare the availability of using different digital commercial non-metric cameras and metric camera observations for architectural building documentation and find the least expensive and available technique with high accuracy for forming three dimensions accurate drawing of historical building in Mansoura city, Egypt. The paper aims also to find the optimum number of observed control points coordinates required on photo for processing and analysis of multiple images. MATLAB programming will be applied for solving least square equations and matrices. Close range Digital Workstation (CDW) program will be applied in this research for processing and analysis of multiple images.

\section{Study area and instruments used}

The site chosen for testing was EL-Nasr Mosque façades in Mansoura city, Egypt. Mansoura city is located on the east bank of the Nile-Damietta branch. It is $120 \mathrm{~km}$ northeast of Cairo. Mansoura is The capital of Dakahlia Governorate which was established by King Kamil Muhammad in 1219 in the era of the Ayyubid state. The studied historical building in this study was EL-Nasr Mosque which was built in 1954 on an area of more than 4.000 square meters. It is located in the center of Al-Jish Street, the main street of Mansoura city, Dakahlia Governorate, Egypt. The mosque is considered the title of Mansoura to receive before heading to the General Office of Dakahlia Governorate and Umm Kulthum Square (Figure 1 and Figure 2).

\section{Instruments used for data collection}

\section{Reflector-less total station}

The cx-103 Total station reflector-less from SOKKIA with accessories was used for measuring the coordinates of specified points in mosque façade containing GCPs (ground control points) and CHPs (check points). The total station has the accuracy of measuring angles equals to $3^{/ /}$and the accuracy of measuring distance was $2 \mathrm{~mm} \pm$ $2 \mathrm{ppm}$ for reflector measuring and $3 \mathrm{~mm} \pm 3 \mathrm{ppm}$ for reflector-less measuring; two tripods; sheet prisms; two prisms of diameter $5 \mathrm{~cm}$ and two foot plates. The used total station was calibrated before completing field observations for the studied research to confirm the specified accuracy for measuring distances and angles.

\section{Metric camera}

There are different types of cameras used in terrestrial photography. All of them may be classified as metric or non-metric. Metric cameras include those cameras which were manufactured for photogrammetric applications. Metric cameras are properly constructed and calibrated before use. Their calibration values for focal length, principal point coordinates and lens distortions can be applied with confidence over long periods of time. Geometric camera calibration is a fundamental pre-requisite for any photographic system that is used to obtain quantitative measurements of the observed scene (Gayde et al., 1997).

The Rollei 6008i film camera was used in this paper. It has the following characteristic: $80 \mathrm{~mm} \mathrm{f/2.8} \mathrm{Hft,} 6 \times 6 / 120$ color film for 12 frames (each frame $6 \times 6 \mathrm{~cm}$ ), SLR (single length Reflex), shutter speed 30 to $1000 \mathrm{sec}$ and Agfadue scanner were used for documenting the monumental building by CRP technique (Rollei, 1993).

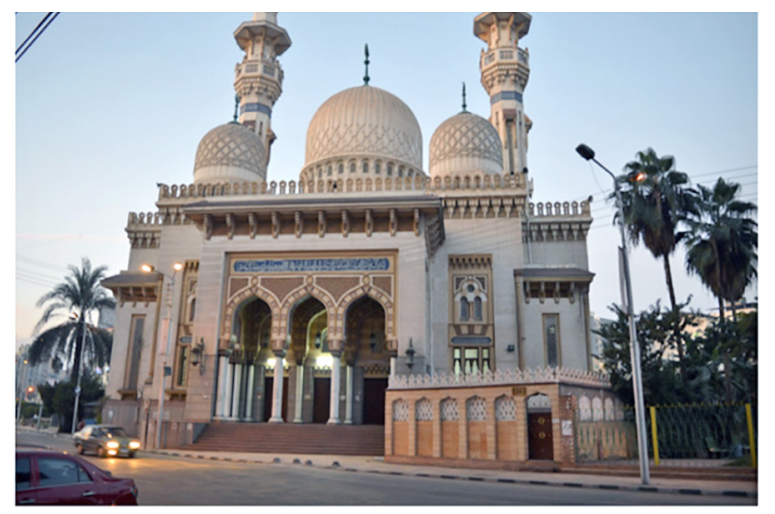

Figure 1. El-Nasr Mosque façade, Mansoura city, Egypt (the studied historical building)

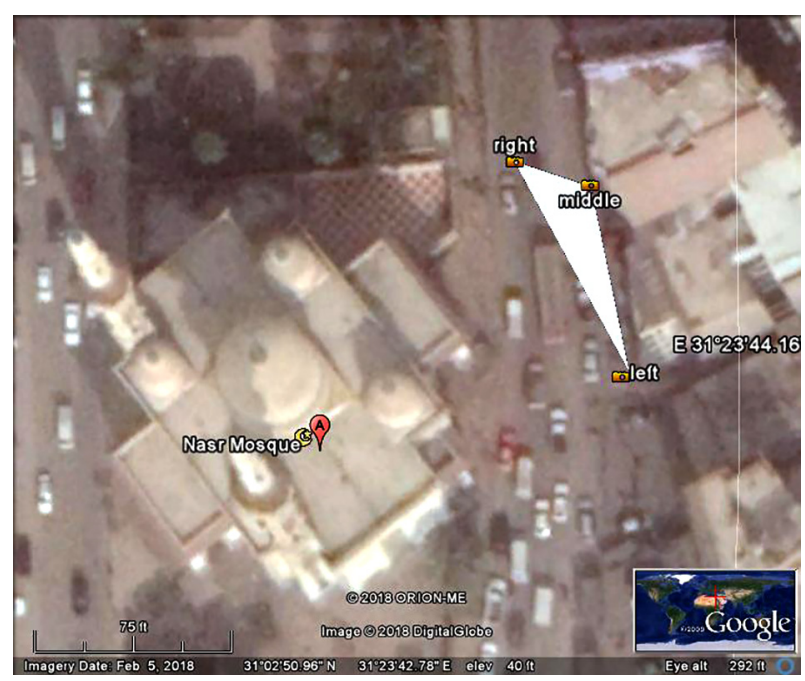

Figure 2. El-Nasr Mosque site from Google Earth image and the occupied positions of photos capture

\section{Digital commercial cameras}

Four types (three digital cameras and one mobile camera) were used as shown in Figure 3 with different resolutions: 
1. Nikon D5200 digital camera with resolution $24 \mathrm{MP}$, sensor size $\left(23.5^{\star} 15.6 \mathrm{~mm}\right)$, resolutions $6000^{\star} 4000$ pixels.

2. Canon SLR digital camera with resolution $18 \mathrm{MP}$, sensor size $22.3^{\star} 14.9 \mathrm{~mm}$.

3. Sony W830 digital camera with resolution $20 \mathrm{MP}$, sensor size (1/2.3") inch.

4. Samsung Galaxy S7 edge mobile camera with resolution 12 MP.

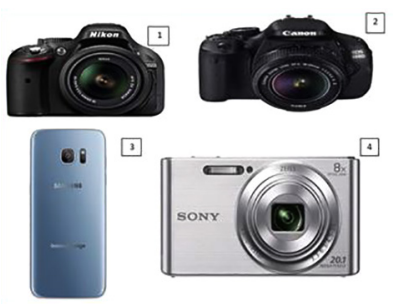

a) Non metric cameras

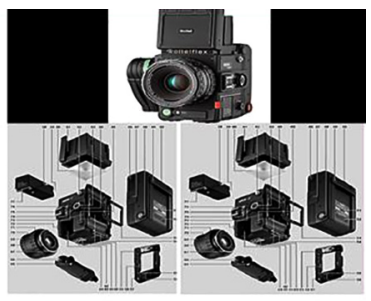

b) Metric camera (Rollei 6008i)
Figure 3. Types of cameras used in the research

\subsection{Field work and data collection}

For drawing and mapping a façade of El-Nasr Mosque with accurate dimensions and details, the technique of close range photogrammetry (CRP) was applied. This technique requires three basic rules which should be followed:

a) Approximately 10-12 clearly identifiable points should be measured in every image. Twenty five control point were used in this research.

b) The image points should be distributed over the whole image (The image points must not be located on a straight line).

c) Every point observed in at least 3 images taken from different exposure stations of the used camera which are fixed in the best possible conditions.

Observations of a number of points on the studied object (mosque façade) using an accurate instrument such as total station instrument were carried out. Some of these points were used as control points and the rest were used as check points to validate the model and processing technique. The steps and procedure of research work are shown in Figure 4.

\subsection{Total station measurements}

Twenty five observed points were selected on the mosque façades at different elevations and different façade surfaces. These points are distributed to provide complete coverage of the mosque façade. These points are located by using sheet prisms of diameter $(1 \mathrm{~cm})$, which are arranged to be visible from the location of the used total station. The coordinates $\left(X_{T S}, Y_{T S}\right.$, and $Z_{T S}$ ) of these points and its accuracy using propagation law were determined and calculated. Figure 5 shows the location and distribution of the observed points on the mosque façade.

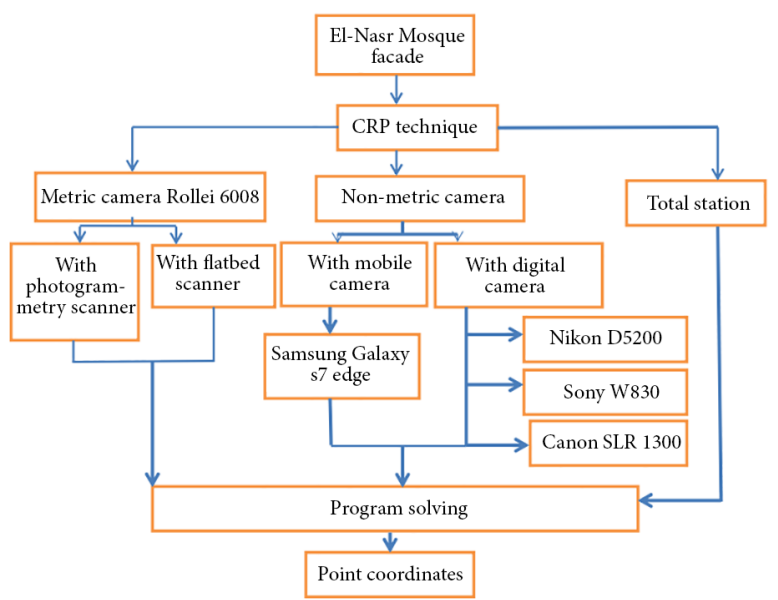

Figure 4. Steps and procedure of research work (field work and data analysis)

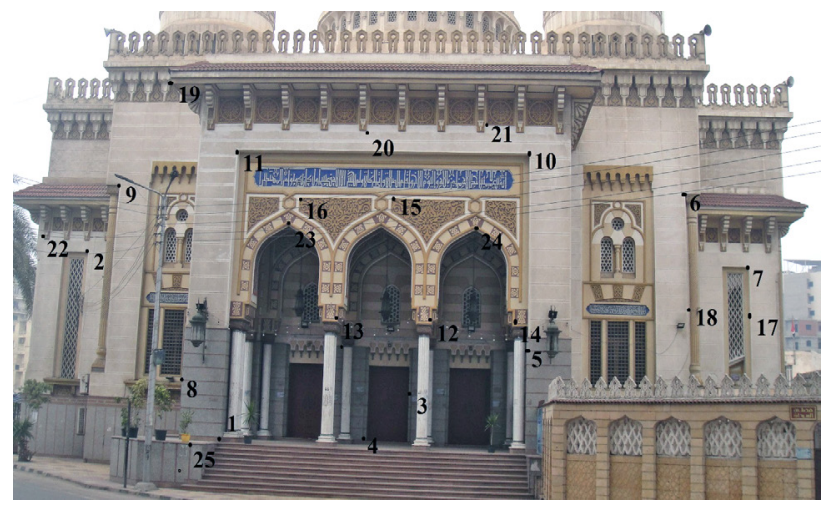

Figure 5. The distribution of observed points on El-Nasr Mosque façade

\subsection{Images capturing}

a) By using metric camera:

Three camera exposure stations were set out in front of the façade as shown in Figure 2. These stations were distributed (left, middle, right) with variable distances from the façade. A hand-held Rollei Metric 6008 normal camera was set up on the planned camera exposure station. The camera was oriented to the line of symmetry of the frontal with the help of the central reseau line. The reseau crosses was directly placed in front of the film plane to allow the numerical corrections of the film deformation. This correction is a decisive criterion for photogrammetric precision evaluation. The camera output was a hard copy format (negative of $60 \times 60 \mathrm{~mm}$ ) as shown in Figure 6 .

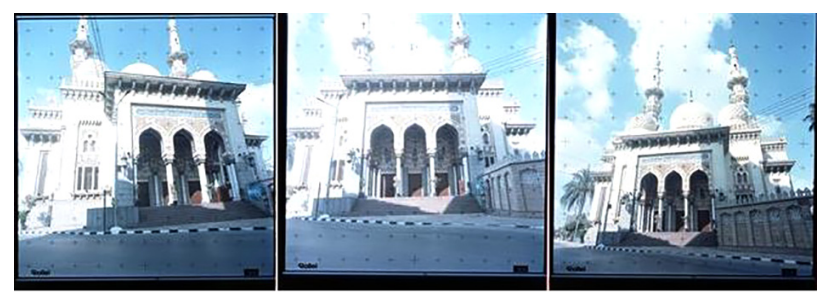

a)

b)

c)

Figure 6. Captured images from three metric camera exposure stations (Rollei 6008 camera) 
b) Transformation from Hard to Soft Copy (Metric camera):

In this research, digital images data were derived from the frame images of photographic film by using two methods. In the first method, the captured images were scanned by using high-precision photogrammetric scanner (AGFA DUE SCAN scanner with resolution $4000 \mathrm{dpi}$, pixel size $6 \mu \mathrm{m})$. In the second method, the captured images were scanned by using normal-precision flatbed scanner (HP Scanjet scanner with resolution $600 \mathrm{dpi}$, pixel size $43 \mu \mathrm{m}$ ).

c) By using non-metric camera:

Three camera exposure stations were set out in the front of mosque façade. These stations were distributed (left, middle, right) with variable distances from the façade (Figure 2). A hand-held Nikon D5200, Sony W830, Canon SLR 1300D, Samsung Galaxy S7 edge mobile digital cameras with $24,20,18,16$ megapixel resolutions respectively were set up on the planned camera exposure stations. The cameras output were a soft copy format image.

\subsection{Software processing}

Close range Digital Workstation (CDW) has two optimized methods for computing multi-photo orientation and bundle adjustment. Multi-photo orientation is a method of recovering the photographic configuration of photo assembly. This method designed for determining camera stations and object points sequentially by intersection and resection model. Rigorous computation is here foregone in favor of high speed. Self-calibration is performed within the process of solving the main problem, for example construction of two or three-dimensional model of the structure. This approach saves time because the required parameters are determined by the same working images (Sementsov et al., 2015).

The calibration of the image recording system relies on how accurate the camera geometry is known. The main parameters of the camera geometry are the principal point and the principal distance. These parameters are estimated simultaneously with the orientation parameters of the network and the object coordinates by the bundle adjustment. Systematic image errors like radial symmetric/asymmetric and tangential lens distortion as well as the affine parameters of the sensor are also estimated in the same process.

Bundle adjustment is a rigorous method of computing and adjusting photo assembly for optimum matching, making allowance for all observations in the object and image space. This method relates each measurement to the desired magnitudes by an observation equation. The system of equations thus obtained contains redundancies and cannot be resolved unequivocally. The result is obtained during adjustment with the purpose of minimizing existing deviations (minimizing the square of error by the least square method).

The program was used for data processing with the operator performing all measurements of control and check points on each image separately and the software calculating a space resection. The control points and check points must be identified in every loaded image to compute the camera exposure station for these images using multi image orientation technique.

Trials in CDW program: Four cases were processed through seven trials in CDW program as shown in Figure 7. Control points had chosen random, covered all the façade and not in straight line are nine. The remaining observed points was used for validation and checking. This processing was applied with all types of cameras either metric or non-metric cameras.

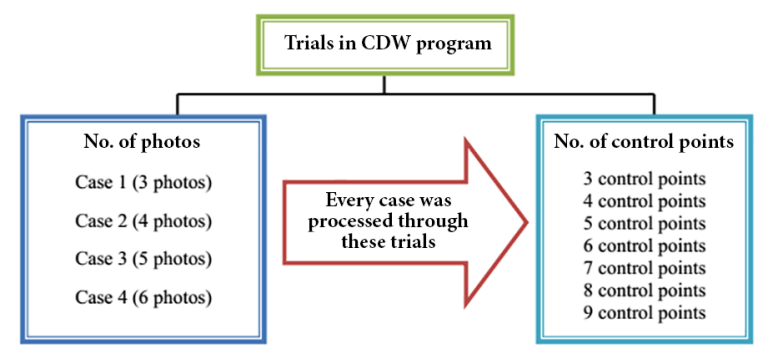

Figure 7. Flow chart of studied trials using CDW program

\subsection{Seven parameter transformation}

When computing the image assembly, the images were oriented to each other via the measured orientation points (assembly points). The camera stations and the object coordinates of the orientation points were obtained in a user defined coordinate system as a result of the fixing system. The functional relation of the bundle adjustment is the central projection which include the parameters of the camera data, the camera stations and the three coordinates $X_{i}, Y_{i}, Z_{i}$ of the object points (Gayde et al., 1997).

Multi-parameter transformation can be applied for relating the perspective center and object coordinates of the perspective center point using the following form:

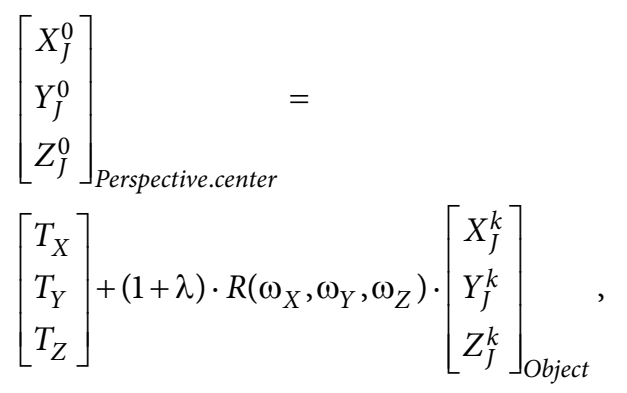

where: $\left[\begin{array}{c}X_{J}^{o} \\ Y_{J}^{o} \\ Z_{J}^{o}\end{array}\right]_{\text {perspectivecenter }}=$ Perspective center coordinates of reference point $J$ at the first epoch of observations; $\left[\begin{array}{c}T_{X} \\ T_{Y} \\ T_{Z}\end{array}\right]=$ translation components vector; $R\left(\omega_{X}, \omega_{Y}, \omega_{Z}\right)=$ 
Rotation matrix; $\left[\begin{array}{c}X_{i}^{p} \\ Y_{i}^{p} \\ Z_{i}^{p}\end{array}\right]_{o b j e c t}=$ The point coordinates in

other observations cycle (object coordinates); $(1+\lambda)=$ The scale factor.

Since the values of rotation components will be too small, the rotation matrix can be simplified and the Equation (1) can be written as:

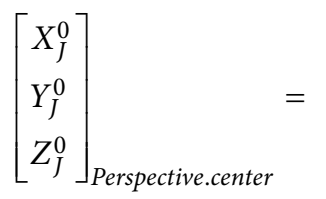

$$
\left[\begin{array}{c}
T_{X} \\
T_{Y} \\
T_{Z}
\end{array}\right]+(1+\lambda) \cdot\left[\begin{array}{ccc}
1 & \omega_{Z} & -\omega_{Y} \\
-\omega_{Z} & 1 & \omega_{X} \\
\omega_{Y} & -\omega_{X} & 1
\end{array}\right]\left[\begin{array}{c}
X_{J}^{p} \\
Y_{J}^{p} \\
Z_{J}^{p}
\end{array}\right]_{\text {Object }}
$$

Equation (2) has seven unknowns (seven parameters of transformation), so for solving this equation you need as minimum 7 equations or coordinates of three points. If the identical points in the two system coordinates are more than three, the least square theory must be applied to find the parameters. The mathematical model for this case (observational least square) will have the form:

$$
\underset{(m, 7)}{A} \cdot \underset{(7,1)}{\delta}+\underset{(m, 1)}{V}=\underset{(m, 1)}{L},
$$

where: $m$ - the number of equations; $n$ - the number of identical points.

The elements of matrix A can be determined by differentiate of Equation (3) with respect to the parameters of transformation.

The weight matrix in this case has dimensions $(\mathrm{m}, \mathrm{m})$ and has the form:

$$
\underset{(12,12)}{W}=\operatorname{diag}\left[\begin{array}{lllll}
\frac{1}{\left(\sigma_{X_{1}}\right)^{2}} & \frac{1}{\left(\sigma_{Y_{1}}\right)^{2}} & \frac{1}{\left(\sigma_{Z_{1}}\right)^{2}} & \cdots & \frac{1}{\left(\sigma_{Z_{m}}\right)^{2}}
\end{array}\right] \text {. (4) }
$$

Then the steps of solving using parametric least squares method were applied to find the seven transformation parameters automatic by CDW software after several trials for different number of control points.

\subsection{Discrepancies of point coordinates}

Finally, the coordinates $(X, Y, Z)$ of the check points were obtained for different cases of control points number from analysis and processing of metric images with flatbed scanner and photogrammetric scanner and from non-metric (digital) images resulted from four used digital cameras using CDW software. These coordinates were compared with the corresponding coordinates obtained by using total station. Then the residual error was calculated, and then the RMSE of the residuals and max residual error of the coordinates were computed.

$$
\begin{aligned}
& e_{\Delta x}=x_{T . S}-x_{M} \& e_{\Delta y}=y_{T . S}-y_{M} \& e_{\Delta z}=z_{T . S}-z_{M} ; \quad(5) \\
& e_{\Delta x}=x_{T . S}-x_{N M} \& e_{\Delta y}=y_{T . S}-y_{N M} \& e_{\Delta z}=z_{T . S}-z_{N M} ;
\end{aligned}
$$

$e_{R}=\sqrt{e_{\Delta x}^{2}+e_{\Delta y}^{2}+e_{\Delta z}^{2}}$

$R M S E=\sqrt{\frac{\sum E^{2}}{n}}$,

where: $x_{M}, y_{M}, z_{M}$-are the coordinates of CHPs (check points) from CRP technique when using metric camera; $x_{N M}, y_{N M}, z_{N M}$ - are the coordinates of CHPs (check points) from CRP technique when using non-metric camera; $x_{T . S}, y_{T S}, z_{T . S}$ - are the coordinates of CHPs from total station; $e_{\Delta x}, e_{\Delta y}, e_{\Delta z}$ - are residual errors of the coordinates; $e_{R}$ - is the resultant residual error; RMSE - is root mean square of residual error; $E$ - residual error of the coordinates in $x, y$ and $z ; n$ - is the number of CHPs.

\section{Results and analysis}

The resulted object coordinates of control points were compared with the corresponding points coordinates obtained from total station observations. Then the residual errors were calculated for all the coordinates. Effect of ground control point's numbers on root mean square of residual error, maximum and minimum point coordinates differences from total station and metric camera with photogrammetry scanner are shown in the Figures 8, 9 and 10.

The number of images taken in the processing was four cases (three, four, five and six images) and the number of control points for fixing system was seven trials (three, four, five, six, seven, and eight, nine control points). It is noted that the resultant residual error changed from $4.87 \mathrm{~mm}$ to $2.39 \mathrm{~mm}$.

The differences of coordinates were decreased if the number of control points and images increase but when eight and nine control points are used, the error was very small.

In this study, according to the result of maximum and minimum differences, the variants eight and nine of control points were the same almost.in term of time, a variable of eight control point with six images was chosen.

The results of RMSE, maximum and minimum differences between the coordinates of check points resulted from total station observations and the corresponding coordinates resulted from imaging processing from metric camera with flatbed scanner and non-metric cameras for the optimum case when using six images and eight control points were shown in Figure 11.

Figure 12 represents the differences in $X, Y$ and $Z$ between the coordinates of check points resulted from total station observations and from all studied camera types for optimum control points number (eight control points) and six images. 
three images

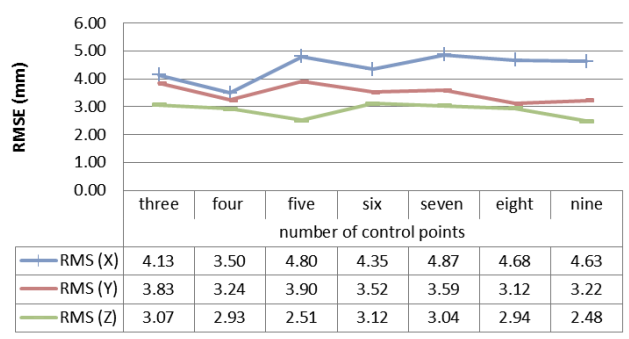

a)

five images

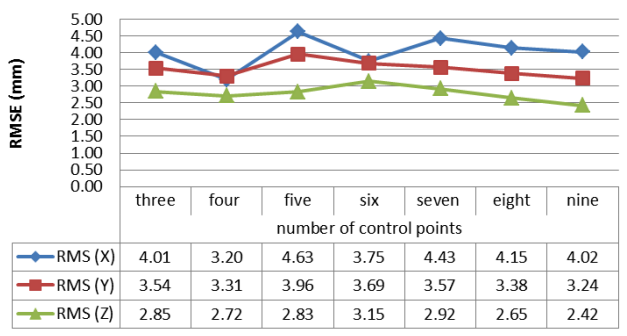

c)

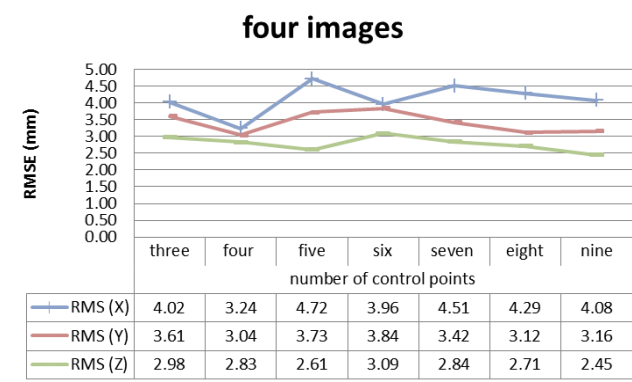

b)

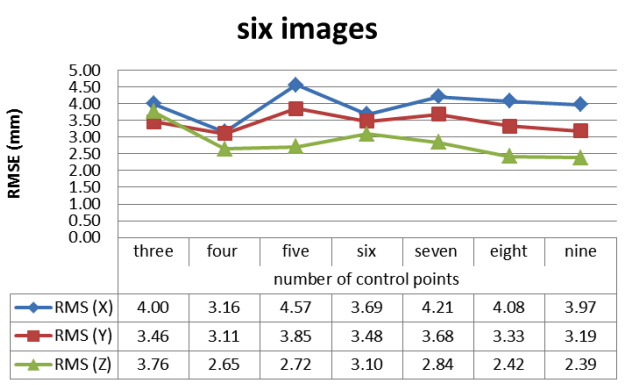

d)

Figure 8. The results of RMS residual errors using metric camera with photogrammetry scanner

\section{three images}

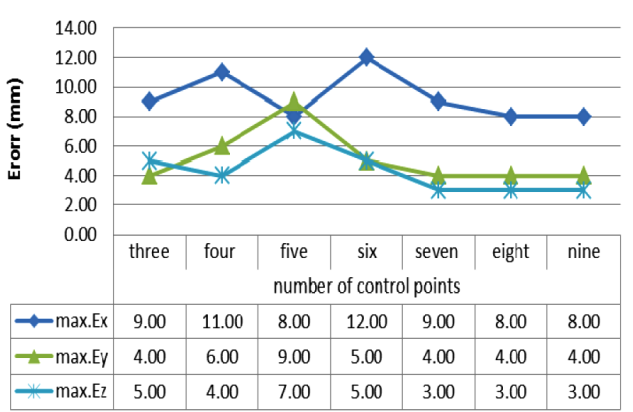

a)

\section{five images}

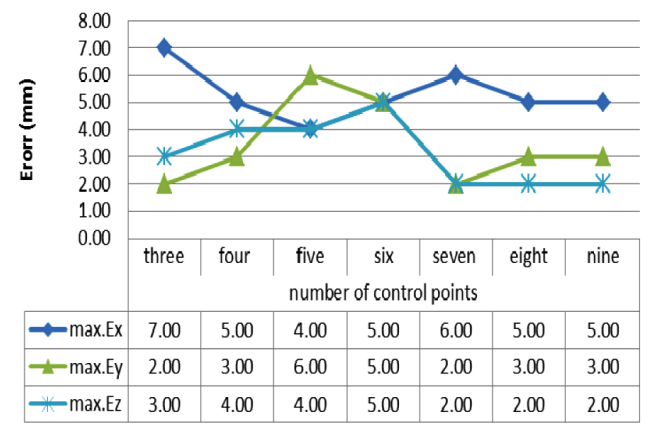

c)

Figure 9. Effect of ground control point's numbers on maximum point coordinates differences between total station observations and metric camera with photogrammetry scanner four images

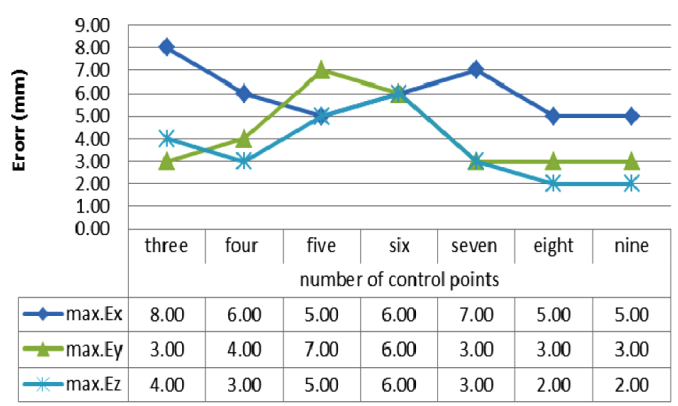

b)

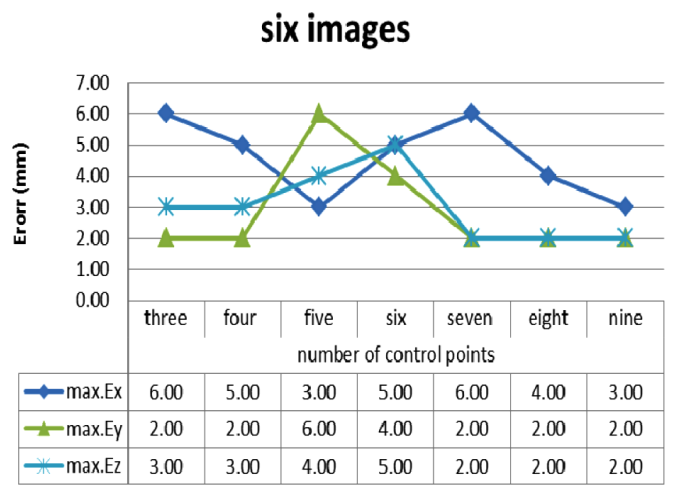

d) 
a)

three images

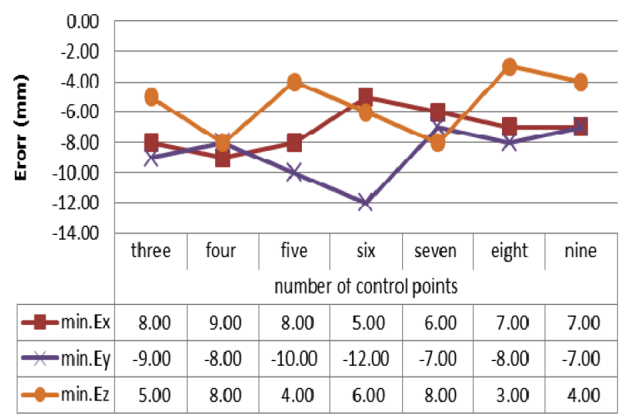

c)

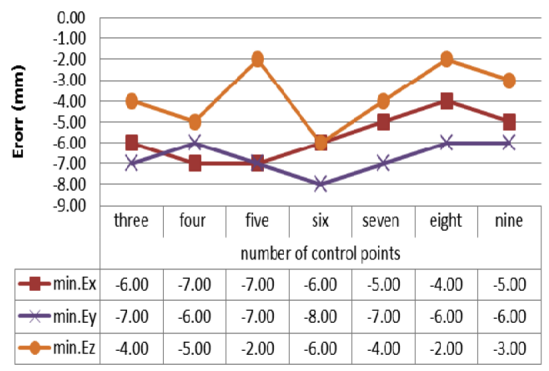

b)

four images

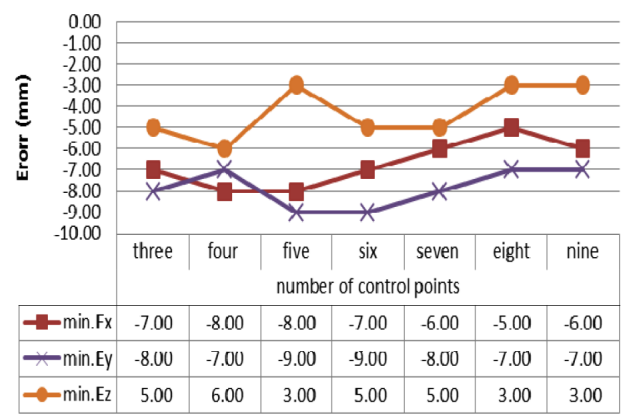

d)

six images

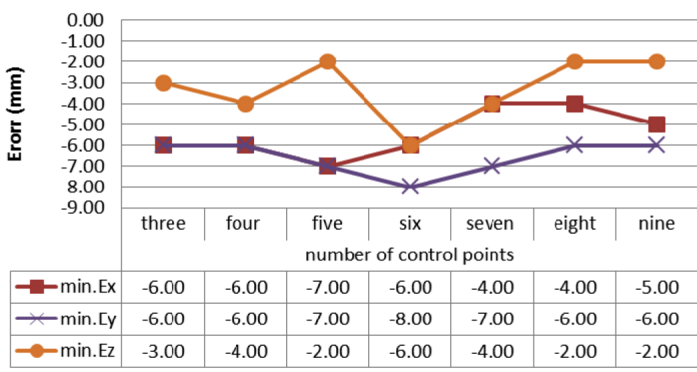

Figure 10. Effect of ground control point's numbers on minimum point coordinates differences between total station observations and metric camera with photogrammetry scanner

a) metric camera with photogrammetry scanner b)

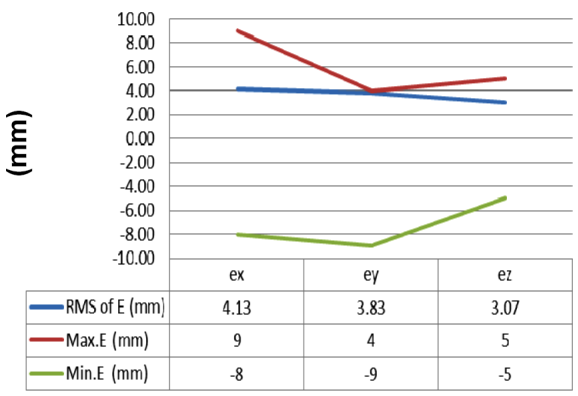

c)

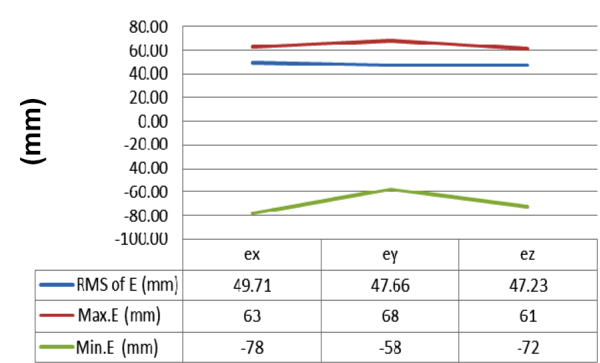

e)

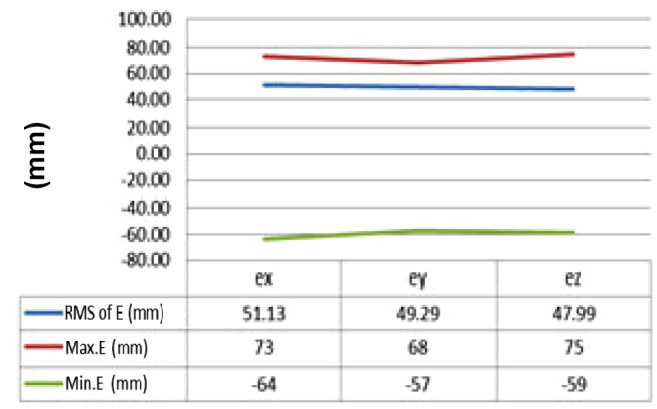

metric camera with flatbed scanner

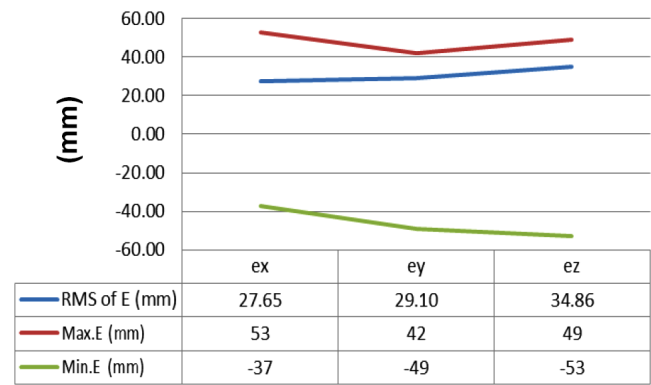

d)

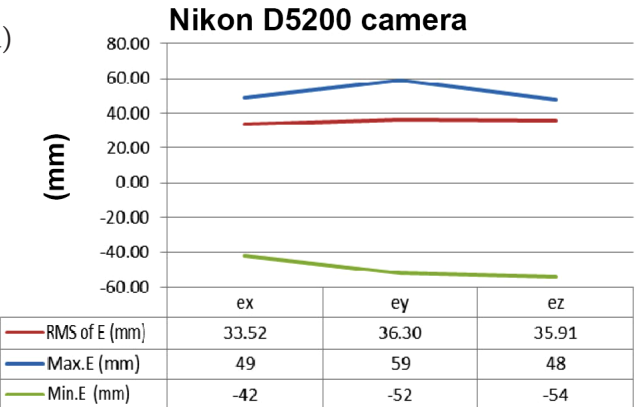

f)

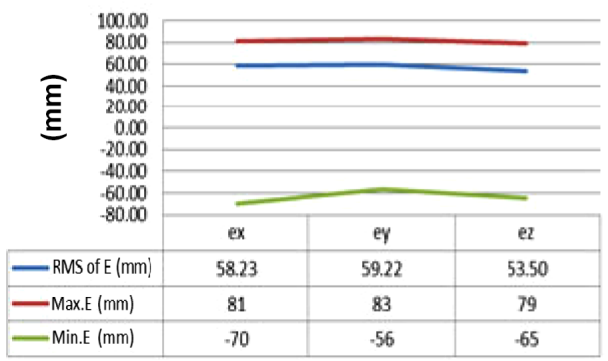

Figure 11. The results of RMSE, maximum and minimum differences between the coordinates of check points resulted from total station and imaging processing from metric camera with flatbed scanner and non-metric cameras 
From the comparison between the accuracy of non metric digital cameras and metric camera with photogrammetry scanner or flatbed scanner in Figure 12, it was noted that the best accuracy result obtained from non metric cameras is digital camera Nikon 5200D with resolution 24 MP where the distortions changed from 41.36 $\mathrm{mm}$ to $59.22 \mathrm{~mm}$ but the differences values for other digital cameras types are higher as shown in Figure 13.

Finally, the reconstructions and mapping of historical site (mosque façade) require high accuracy measuring device like metric camera with photogrammetry scanner. Form the data available (coordinates of points) and image processing after multi-photo and bundle adjustment using Close range Digital Workstation (CDW) program the façade of El-Nasr Mosque can be accurately drawn as shown in Figure 14.

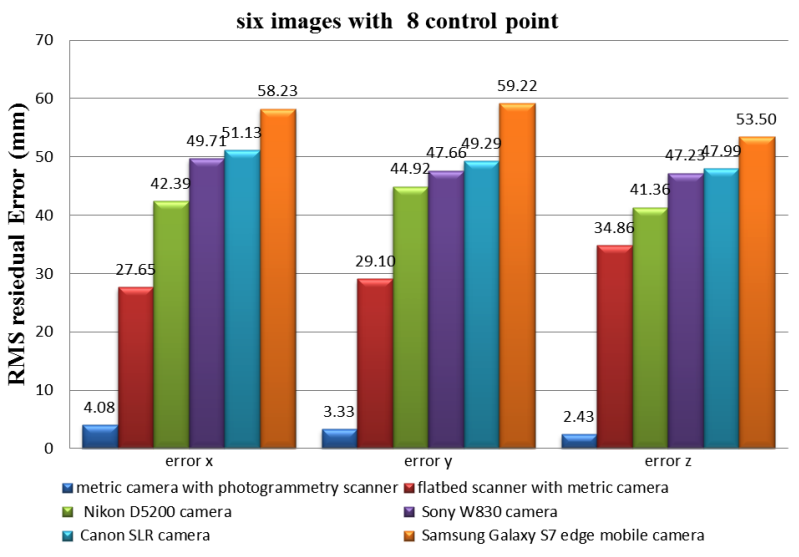

Figure 13. RMS residual errors in CRP technique for all types of cameras using six images and eight control points

a)

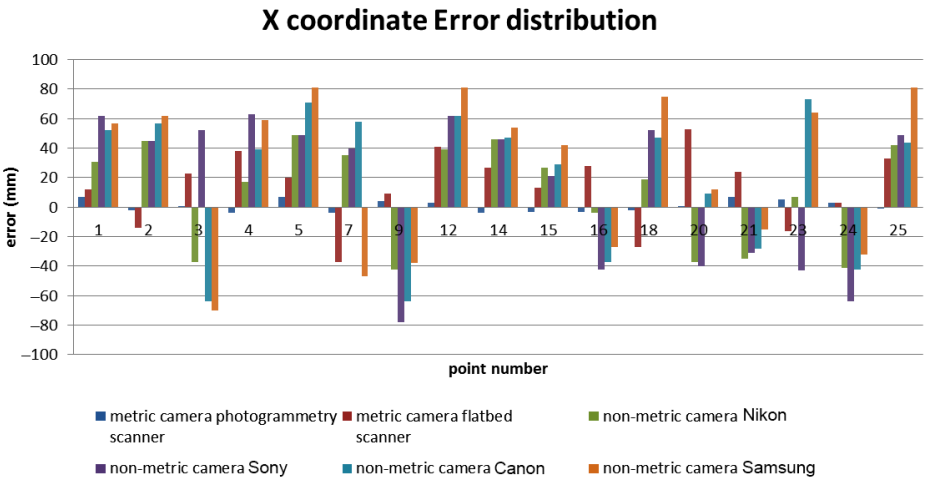

b)

Y coordinate Error distribution

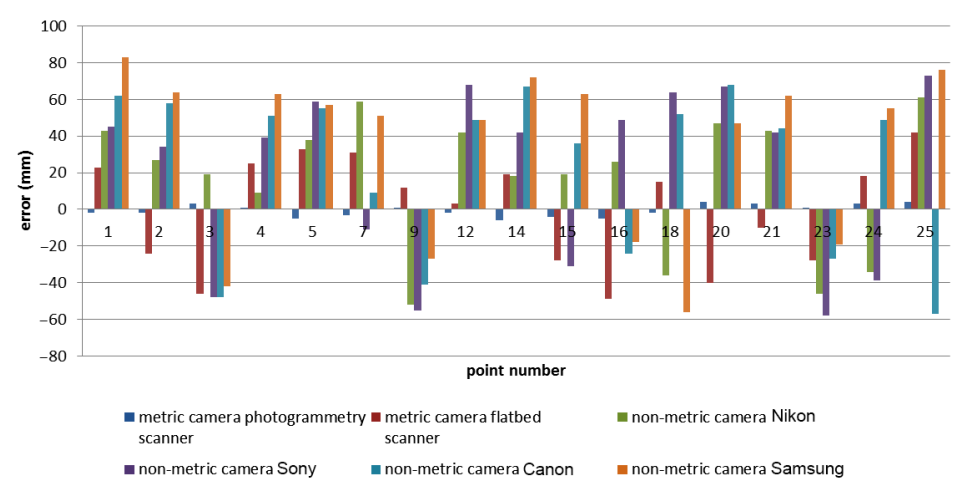

c)

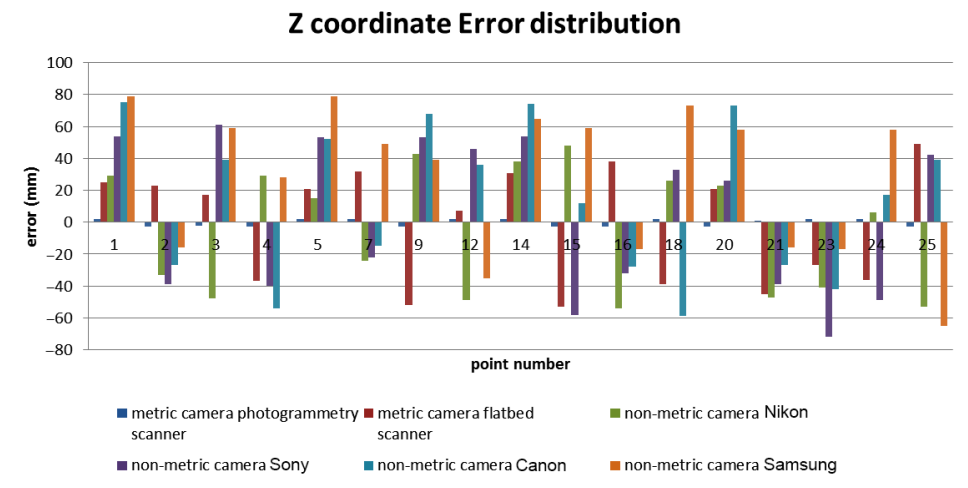

Figure 12. Differences of check points in $X, Y, Z$ axis between total station observations and coordinates from metric and non-metric cameras 


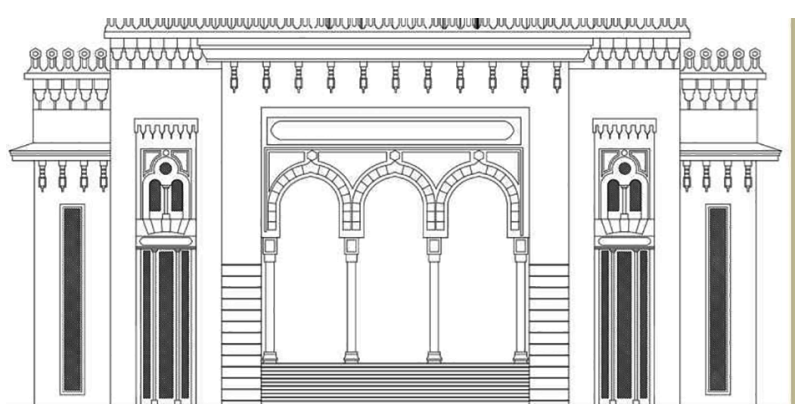

Figure 14. 2D drawing of El-Nasr Mosque façade depending on resulted image processing

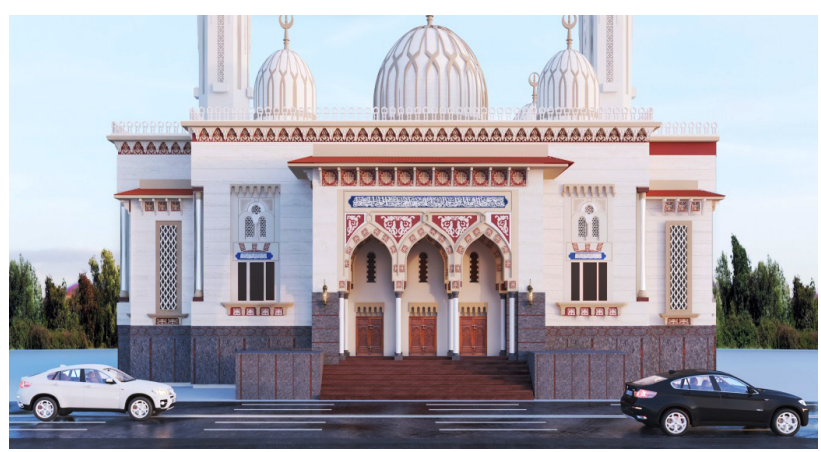

Figure 15. 3D model of the façade with surface rendering

Surface rendering, which involves the generation of a 3D façade model with real world surface texture, is constructed in Figure 15. 3D model surfaces were added by surface texture to give a real world appearance to the display model.

\section{Conclusions}

The objective of the current paper is to study, compare and asses the use of close range photogrammetry (CRP) technique and commercial digital cameras to reconstruct views for historical building. Close range photogrammetry technique was applied using metric and non-metric (digital) cameras. The metric camera was used with photogrammetric scanner and flatbed scanner. The façade of Nasr mosque in Mansoura city, Egypt was selected for application in this study. The following conclusions can be drawn:

1. Commercial inexpensive digital or mobile cameras can be readily applied to photogrammetric measurement and civil engineering applications. Non metric (digital) cameras with CRP technique product different accuracy that depended on the resolutions of cameras and camera specifications. If the camera resolution is high, the percentage of error decreases and consequently the accuracy increases. Such results showed large errors which discard the possibility of using low resolution non metric camera for $3 \mathrm{D}$ restoration applications.

2. Using photogrammetry scanner with metric camera instead of flatbed scanner in close range photogrammetry technique is accurate enough to be beneficial in $3 \mathrm{D}$ architectural building documentation. The results are indicated that flatbed scanner with metric camera was acceptable in limited cases that RMSE was $46.7 \mathrm{~mm}$.
3. According to the studied cases of control points number and images number and its effect on the discrepancies of check points coordinates, it is found that using six images for façade with eight control points is the optimum conditions for getting the accurate mapping of mosque façade.

4. Close range Digital Workstation (CDW) software is dealing with digital image processing and has the ability of determining the coordinates of points from photo precisely.

\section{Acknowledgements}

The authors would like to thank Dr. Ateya Bekheet, Ph. D. degree, researcher in National Authority for Remote Sensing \& Space Sciences for his helping in experimental works and programming.

\section{References}

Abed, F. M., Mohammed, M. U., \& Kadhim, S. J. (2017). Architectural and cultural heritage conservation using low-cost cameras. Applied Research Journal, 3, 376-384.

Cardenal, J., Mata, E., Castro, P., Delgado, J., Hernandez, M. A., Perez, J. L., Ramos, M., \& Torres, M. (2004). Evaluation of a digital non metric camera (Canon D30) for the photogrammetric recording of historical buildings. The International Archives of the Photogrammetry, Remote Sensing and Spatial Information Sciences, 34(Part XXX).

Elsharkawy, A. (2017). Accuracy of modern three dimensional data acquisition system for the restoration of historical buildings [Doctoral thesis]. Faculty of Engineering, Port Said University.

Gayde, J. C., Humbertclaude, C., \& Lasseur, C. (1997). Prospects of close range digital photogrammetry in large physics installations. In Proceedings of the 5th International Workshop on Accelerator Alignment (pp. 1-12), Chicago, USA.

Khalaf, A., Ataiwe, T., Mohammed, I., \& Kareem, A. (2018). 3D Digital modeling for archeology using close range photogrammetry. MATEC Web of Conferences, 162, 03027. https://doi.org/10.1051/matecconf/201816203027

Luhmann, T. (2010). Close range photogrammetry for industrial applications. ISPRS Journal of Photogrammetry and Remote Sensing, 65(6), 558-569.

https://doi.org/10.1016/j.isprsjprs.2010.06.003

Markiewicz, J., Łapiński, S., Pilarska, M., Bieńkowski, R., \& Kaliszewska, A. (2018). Investigation into the use of action cameras in the documentation of architectural details-the case study of a baroque chamber. The International Archives of the Photogrammetry, Remote Sensing and Spatial Information Sciences, 42(2), 667-674.

https://doi.org/10.5194/isprs-archives-XLII-2-667-2018

Rollei. (1993). Rollei-flex6008 user's manual. Rollei Foto-Technic $\mathrm{GmbH} \& \mathrm{Co}$ KG.

Sementsov, A. V., Nikitin, V. N., \& Chermoshentsev, A. Yu. (2015, March 23-24). 3D object modeling from images taken by non-metric digital cameras: problems and their solutions. In $2^{\text {nd }}$ International Workshop on "Integration of Point- and Area-Wise Geodetic Monitoring for Structures and Natural Objects" (pp. 13-15), Stuttgart, Germany.

Suveg, I., \& Vosselman, G. (2000). 3D reconstruction of building models. International Archives of Photogrammetry and Remote Sensing, 33, 538-545. 